\title{
A multi-technique approach for characterizing the SVN49 signal anomaly, part 1: receiver tracking and IQ constellation
}

\author{
André Hauschild • Oliver Montenbruck • \\ Steffen Thoelert · Stefan Erker • Michael Meurer • \\ Javad Ashjaee
}

Received: 9 November 2010/Accepted: 8 January 2011/Published online: 4 February 2011

(C) The Author(s) 2011. This article is published with open access at Springerlink.com

\begin{abstract}
A characterization of the signal anomaly of SVN49 is presented. A mathematical model is developed to relate the observed multipath to the internal signal reflection. The analyses provided are based on measurements, which have been collected during a dedicated tracking campaign with a 30-m dish antenna. Data on the L1 and L2 frequency have been collected with four different receivers. In addition, IQ samples have been recorded directly with a spectrum analyzer. The multipath combination of the receiver measurements on L1 and L2 is analyzed to demonstrate the effect of the signal reflections on different correlator spacing. The capability to suppress the signal reflection with receiver multipath mitigation methods is demonstrated. Finally, preliminary estimates of the attenuation, delay, and phase shift over elevation are obtained from an IQ sample analysis.
\end{abstract}

Keywords Multipath - SVN49 - PRN1 - IQ sampling · E-L correlator

Disclaimer: The activities have been funded within the frame of DLR's Center for Excellence for Satellite Navigation. The choice of GNSS receivers and other test equipment employed for the SVN49 signal analysis is based on availability at the time of the test. Neither DLR nor the GPS Wing endorse or recommend any specific commercial product.

A. Hauschild · O. Montenbruck $(\square)$

DLR/GSOC, Wessling, Germany

e-mail: oliver.montenbruck@dlr.de

S. Thoelert $\cdot$ S. Erker $\cdot$ M. Meurer

DLR/IKN, Wessling, Germany

J. Ashjaee

JAVAD GNSS, Moscow, Russia

\section{Introduction}

SVN49 stands out of the other satellites of the GPS constellation. This space vehicle, which is currently assigned to PRN 1, is a Block IIR-M satellite and was launched on March 24, 2009, as the second-last of its type. The satellite carries an experimental signal generation payload for the transmission of the L5 signal. The implementation of this feature, which was not foreseen for normal Block IIR-M satellites, became necessary to meet a deadline in frequency utilization set by the International Telecommunication Union (ITU) (Erwin et al. 2009).

After the activation of the signal transmissions, users experienced unexpected residuals of the pseudorange observations on L1 and L2 (Gao et al. 2009; Meurer et al. 2009; Springer and Dilssner 2009). It turned out that the satellite is affected by a signal anomaly and transmits an undesired internal reflection of the L1 and L2 signals in addition to the direct signals. This reflection is created at a filter of the L5 signal generation unit. The impact of this phenomenon on the position and time estimation has been assessed by El-Arini et al. (2010). Komjathy et al. (2010) discuss the effect on group delay and ionosphere slant delay estimation. However, additional studies are expedient for a mathematical description of the problem and the development of a multipath model. This model will help to assess the impact of the signal reflection on the user community.

A tracking campaign to characterize the effect using different techniques has been performed jointly by German Aerospace Center (DLR) and the GPS Wing using DLR's 30-m dish antenna in Weilheim. We summarize the key findings of the campaign in two parts: The first part introduces the mathematical multipath model and the experiment setup for the tracking campaign. An overview of tracking results with four different receivers connected to 
the high-gain antenna is provided. This setup allows studying the impact of the signal reflection on different correlator settings. Additionally, the effect of multipath mitigation techniques, implemented in the different receivers, has been tested. Furthermore, results of the analysis of IQ samples are presented, which allows the estimation of characteristic properties like delay, attenuation, and phase of the satellite-induced multipath component of SVN49.

A companion paper will include the reconstruction of the chip shape based on the IQ samples. The multipath model for the reflected signal from SVN49 will be parameterized based on these analyses. For validation, the results of the model will be compared to the receiver tracking results from the first part of the paper.

\section{Description and modeling of the multipath reflection}

Figure 1 provides an overview of the transmission chain of the direct and reflected signal for SVN49. The core part is the antenna coupler network, which has the signal generation unit for the L1 and L2 signals connected to the first input port J1. The experimental payload for L5 is connected to the secondary port $\mathrm{J} 2$. Part of the signal fed into the $\mathrm{J} 1$ port of the antenna coupler leaks out of the $\mathrm{J} 2$ port. It enters the cable connecting the L5 signal generation unit, is reflected at the L5 filter, and then fed back into the antenna via the second port with an additional geometric cable length of approximately $8 \mathrm{~m}$ (Lake and Stansell 2009). The direct signal at the $\mathrm{J} 1$ port $\mathrm{S}_{\mathrm{J} 1}$ and the reflected signal, which is attenuated, delayed, and shifted in phase, at the $\mathbf{J} 2$ port $\mathrm{S}_{\mathrm{J} 2}$ are illustrated in Fig. 1.

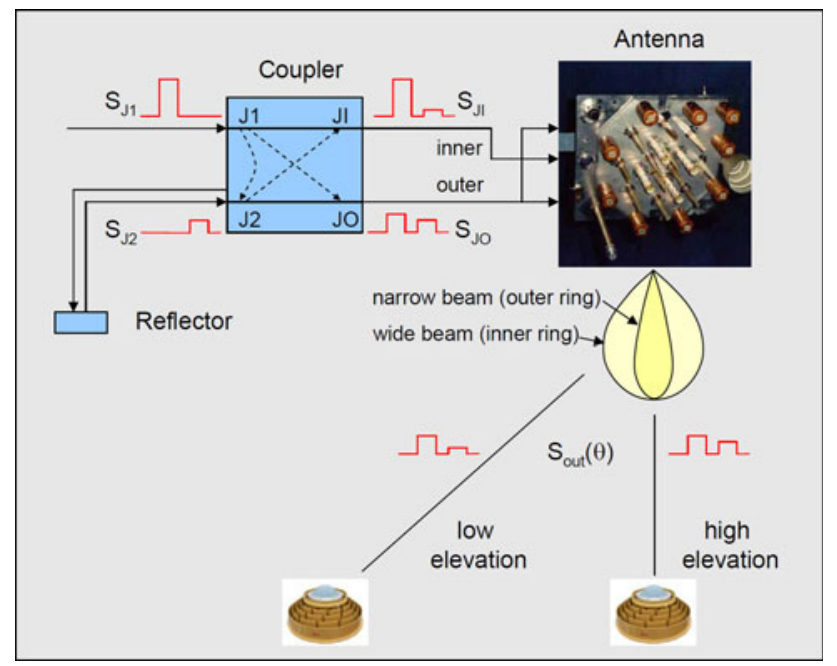

Fig. 1 Schematic of the signal transmission chain of the direct and reflected signal on SVN49
The coupler network distributes the signal power to the twelve antenna elements, which are grouped in two rings: an inner ring with four and an outer ring with eight elements. The inner ring transmits most of the power from the first port of the coupler network with a broad pattern. The outer ring transmits the signal with lower power in a focused pattern and with different phase. As a result, the signal power at small boresight angles (or equivalently high elevation angles on the earth's surface) is reduced in favor of a more uniform distribution for users at high and low boresight angles from the satellite's perspective. The second input port $\mathrm{J} 2$ of the coupler network has a reversed power distribution compared to the first port, i.e. more of the power goes to the outer ring with the higher focus and less is transmitted via the inner ring (Lake and Stansell 2009). As a result, the reflections of the L1 and L2 signals are attenuated and superimposed with the direct signals at the output port for the inner antenna ring JI as illustrated in Fig. 1. Vice versa, at the output port for the outer ring the attenuated direct signal is superimposed with the reflected signal.

Finally, the user receives a signal $S_{\text {OUT, }}$ which is a combination of $S_{\mathrm{JI}}$ and $S_{\mathrm{JO}}$ and exhibits a dependency on the boresight angle $\theta$ or, equivalently, the elevation angle of SVN49. As a result, the reflected signal manifests itself at the user's receiver as a multipath error, which has a dependency on elevation (Langley 2009).

For the characterization of the signal anomaly, a mathematical model shall be developed in the following. The direct signal $S_{\mathrm{J} 1}$, which is fed into the primary connector of the antenna coupler, is written as follows:

$S_{\mathrm{J} 1}=A(t) e^{j \omega t}$

In this equation, $A(t)$ is the time-dependent amplitude of the signal, $\omega$ is the angular frequency, and $t$ is the time. The reflected signal, which is fed back into the secondary port $\mathrm{J} 2$, is attenuated by a factor $\alpha_{\mathrm{R}}$, delayed by a time-constant $\tau_{\mathrm{R}}$, and shifted by a phase shift $\varphi_{\mathrm{R}}$ :

$S_{\mathrm{J} 2}=\alpha_{\mathrm{R}} A\left(t-\tau_{\mathrm{R}}\right) e^{j\left(\omega t+\varphi_{\mathrm{R}}\right)}$

The received signal $S_{\text {OUT }}$ is a superposition of the two previous input signals. It is important to consider that both signals are affected by the antenna coupler network and the transmitting antenna. Thus, $S_{\text {OUT }}$ depends on the transfer functions $H_{\mathrm{J} 1}$ and $H_{\mathrm{J} 2}$ from the primary and the secondary input port to the user antenna on the ground:

$S_{\text {OUT }}=H_{\mathrm{J} 1}(\theta) S_{\mathrm{J} 1}+H_{\mathrm{J} 2}(\theta) S_{\mathrm{J} 2}$

$H_{\mathrm{J} 1}$ and $H_{\mathrm{J} 2}$ are not constant but have a dependency on the boresight angle $\theta$. The transfer functions can also be expressed as complex exponential functions:

$H_{\mathrm{J} 1}=h_{\mathrm{J} 1}(\theta) e^{j \varphi_{\mathrm{J} 1}(\theta)}$ 
$H_{\mathrm{J} 2}=h_{\mathrm{J} 2}(\theta) e^{j \varphi_{\mathrm{J} 2}(\theta)}$

In (4a) and (4b), $h_{\mathrm{J} 1}$ and $h_{\mathrm{J} 2}$ describe the gain for signals from the primary and secondary input port, respectively, depending on the boresight angle. Similarly, $\varphi_{\mathrm{J} 1}$ and $\varphi_{\mathrm{J} 2}$ denote the boresight-dependent phase shifts of the input signals. It is assumed that $H_{\mathrm{J} 1}$ and $H_{\mathrm{J} 2}$ do not cause a differential delay of the signal. This assumption is based on the reasoning that a delay between the signal of the inner and outer antenna ring would cause a boresight-dependent (or elevation-dependent) group delay of the received signals, which is not observed for other GPS satellites. Combining (1)-(4b), we find the following expression for $S_{\text {OUT: }}$

$$
\begin{aligned}
S_{\mathrm{OUT}}= & h_{\mathrm{J} 1}(\theta) A(t) e^{j\left(\omega t+\varphi_{\mathrm{J} 1}(\theta)\right)} \\
& +h_{\mathrm{J} 2}(\theta) \alpha_{\mathrm{R}} A\left(t-\tau_{\mathrm{R}}\right) e^{j\left(\omega t+\varphi_{\mathrm{R}}+\varphi_{\mathrm{J} 2}(\theta)\right)}
\end{aligned}
$$

The delay $\tau$, the attenuation $\alpha$, and the phaseshift $\varphi$ of the reflected signal at the user's end of the signal transmission chain can then be related to the corresponding values $\alpha_{R}$, $\tau_{\mathrm{R}}$, and $\varphi_{\mathrm{R}}$ of the reflection inside the satellite:

$\tau=\tau_{\mathrm{R}}$

$\varphi=\varphi_{\mathrm{R}}+\varphi_{\mathrm{J} 2}(\theta)-\varphi_{\mathrm{J} 1}(\theta)$

$\alpha=\alpha_{\mathrm{R}} h_{\mathrm{J} 2}(\theta) / h_{\mathrm{J} 1}(\theta)$

Measurements of the antenna gain and phase shift for the L1 frequency as functions of the boresight angle have been reported by Ericson et al. (2010), which allows the determination of the characteristic parameters of the internal reflection based on the observed signal on L1.

\section{Experiment setup}

SVN49 has been tracked using the 30-m dish antenna located at DLR's ground station in Weilheim, Germany, during a campaign on April 8-19, 2010. The signal analysis facility installed in the deep space antenna is routinely used for tests and performance analyses of GNSS satellites (Thoelert et al. 2009a). Due to the small beam width of $0.5^{\circ}$, the signals of a single satellite can be tracked with significantly higher gain compared to normal GNSS antennas. Furthermore, multipath reception from ground based reflectors is almost entirely avoided. Thus, apart from signal delays in the ionosphere and troposphere, the measurements are only affected by receiver noise.

The time of the tracking campaign has been coordinated to coincide with a series of high-elevation passes over the tracking antenna in Weilheim. At that point in time, SVN49 was moved to a new slot in the GPS constellation in the course of the repositioning campaign to achieve better global coverage. This gradual change in the orbit shifted the satellite's ground track over the antenna location from East to West, resulting in a zenith pass on April 15. As a result, SNV49 could be tracked for more than one week with maximum elevations higher than $89^{\circ}$, which provided a unique opportunity for signal analysis of the signals over the complete range of elevations. The M-code of SVN49 had been deactivated exclusively for this tracking campaign during April 13-20, 2010. Furthermore, the satellite transmitted unencrypted P-code during this period.

The setup of the experiment is depicted in Fig. 2. The measurement equipment is connected to a specially designed antenna feed, which is optimized for the reception of navigation signals in the L-band. IQ samples of the signals are recorded directly using a spectrum analyzer. For this particular tracking campaign, two Agilent Spectrum Analyzers are used to record samples with a length of $1 \mathrm{~s}$ every $15 \mathrm{~min}$ and $100 \mathrm{~ms}$ every $100 \mathrm{~s}$.

Additionally, four different receivers are connected to the feed via a passive 4-way signal splitter. A Javad Delta TRE-G3TH (or DG3TH), a NovAtel OEMV, a Septentrio PolaRx2, and a NavCom SF3050 receiver are used. The receivers' proprietary raw measurements are recorded and then converted into Rinex files, prior to further processing.

All receivers except for the PolaRx 2 were operated with dedicated firmware versions, which have been especially designed by the corresponding manufacturers for this tracking campaign. The receivers are configured to use conventional early-minus-late (E-L) correlators for most of the tracking campaign. After the zenith pass, the multipath mitigation techniques implemented in the different receiver are activated. The DG3TH receiver tracks the C/A, L2C, $\mathrm{P} 1$, and P2 signals of SVN49 on ten different channels with early-minus-late correlator spacing varying from 0.1 to 1.0 chip. For the tests with the internal multipath mitigation,

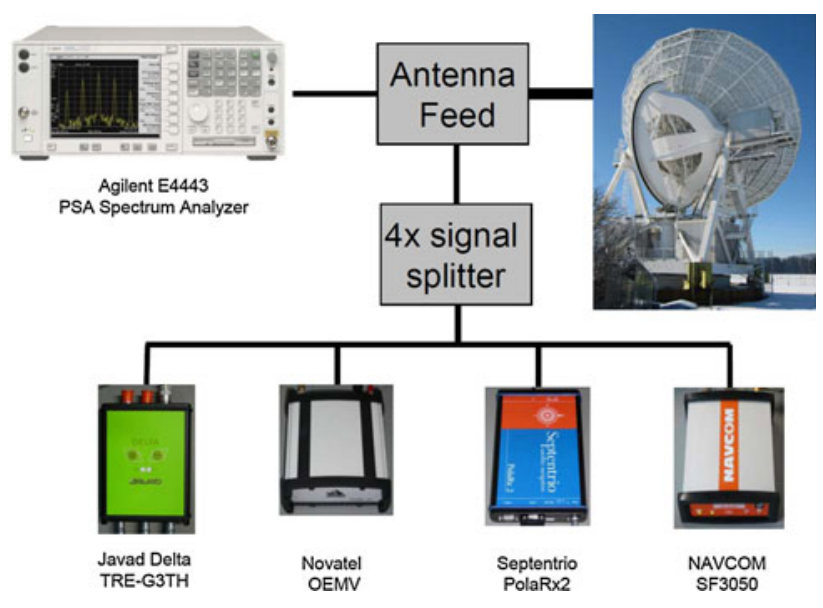

Fig. 2 Experiment setup for SVN49 tracking campaign 
Table 1 Overview of receivers and correlators used in the tracking campaign

\begin{tabular}{|c|c|c|c|}
\hline \multirow[t]{2}{*}{ Receiver } & \multirow[t]{2}{*}{ Firmware } & \multicolumn{2}{|l|}{ Correlator settings } \\
\hline & & Conventional tracking & Multipath mitigation \\
\hline Javad Delta TRE-G3TH & 3.2.0b3_tstprn1 & $0.1 \ldots 1.0$ chip E-L & mpnew (standard firmware $3.1 .5 \mathrm{~b} 1$ ) \\
\hline NovAtel OEMV & $3.700 \mathrm{~S} 30$ & 1.0 chip E-L & PAC \\
\hline NavCom SF3050 & SVN49testmode & - & Hatch-correlator \\
\hline Septentrio PolaRx2 & $2.6 .0-\mathrm{dln} 1$ & $\begin{array}{l}\text { C/A: } 1 / 30 \text { chip E-L } \\
P(Y): 2 / 3 \text { chip E-L }\end{array}$ & APME \\
\hline
\end{tabular}

the standard firmware version 3.1.5b1 has been used. The OEMV receiver tracks $\mathrm{C} / \mathrm{A}$ and $\mathrm{L} 2 \mathrm{C}$ with a conventional 1.0 chip E-L correlator. The pulse aperture correlator (PAC) is used for the tests with multipath mitigation (Jones et al. 2004). In this test, the receiver was also configured to track P2 signals. The SF3050 receiver tracks GPS C/A, P1, L2C, and P2 signals with a Hatch-correlator, which offers improved multipath resistance compared to conventional correlators (Hatch et al. 2007). Finally, the PolaRx2 receiver is operated with a standard firmware for the entire period. The receiver provides $\mathrm{C} / \mathrm{A}$-code measurements based on a 1/30 chip early-late correlator for C/A-code. For $\mathrm{P} 1$ and $\mathrm{P} 2$, the correlator spacing is $2 / 3$ of a chip. The "A Posteriori Multipath Estimator" (APME) is activated for tracking with multipath mitigation (Sleewaegen and Boom 2001). The receiver information is summarized in Table 1. Even though some of the receivers support tracking of the L5 signal as well, the analysis in this paper is limited to the signals on L1 and L2.

\section{Receiver measurements with a high-gain antenna}

Prior to the discussion of the tracking results, the processing and analysis strategy shall be explained here in further details. As already mentioned, the raw measurements of each receiver are converted into Rinex files. Next, the $1 \mathrm{~Hz}$ observations are smoothed with a Hatch filter with $50 \mathrm{~s}$ smoothing interval and decimated to $10 \mathrm{~s}$ steps. The pre-processing step has been done for each receiver irrespective of the fact that some receivers have already applied internal smoothing. The combined effect of pseudorange multipath and receiver noise on a signal can be assessed from the difference of code and carrier phase observations from the corresponding signal, which is corrected for the ionospheric delay using a carrier phase measurement from a second frequency. This combination is generally referred to as the multipath combination and implies the assumption that the carrier phase multipath is negligible compared to the pseudorange multipath. The multipath combination for a single epoch can be computed from (Kee and Parkinson 1994):
$\operatorname{MP}\left(\rho_{\mathrm{A}}\right)=\rho_{\mathrm{A}}-\Phi_{\mathrm{A}}-2 \frac{f_{\mathrm{B}}^{2}}{f_{\mathrm{A}}^{2}-f_{\mathrm{B}}^{2}}\left(\Phi_{\mathrm{A}}-\Phi_{\mathrm{B}}\right)+b_{\mathrm{A}}$

In this equation, $\mathrm{A}$ and $\mathrm{B}$ are the two signals involved, and $f_{\mathrm{A}}$ and $f_{\mathrm{B}}$ are the corresponding frequencies. The pseudorange and carrier phase observables denote $\rho$ and $\Phi$, respectively. The result of the equation depends on the pseudorange multipath errors and receiver noise, but is also offset by an arbitrary bias $b$ due to the carrier phase ambiguities and code delays. To achieve comparable results for the different receivers, this arbitrary offset must be removed in a consistent manner. According to Lake and Stansell (2009), the antenna gain pattern for signals fed into the secondary port of the antenna coupler has a null at an elevation angle of approximately $40^{\circ}$, and the phase pattern reverses its polarity at this point. For L2, the null in the gain pattern of the secondary port appears at approximately $30^{\circ}$. Therefore, the multipath plots for L1 and L2 have been aligned to zero at $40^{\circ}$ and $30^{\circ}$ elevations, respectively.

In addition to the alignment of the offset, a second correction has been applied to remove a trend in the multipath combination over time, which appears to be linear to first approximation. This slope was found to be present in data from all receivers and has a magnitude of a few centimeters per hour. The effect corresponds to a divergence of the pseudorange and carrier phase over time and leads to a mismatch of the ascending and descending part of the multipath curve if plotted over elevation. This effect could not only be observed for SVN49, but also for SVN57, a Block IIR-M satellite launched in December 2008, which has been tracked as a reference. This divergence is usually not visible in the multipath combination with data from a normal GNSS antenna. A conclusive explanation for this phenomenon has not been found so far. The slope shows a significant variation from day to day. The DG3TH, OEMV, and SF3050 receivers seem to exhibit similar slopes on the same days, whereas the PolaRx2 receiver differs significantly. Code or phase delay variations in the receiver or the transmitter (or a combination of both) are a possible explanation for this effect. It should be noted that code and phase variations of delays 
common for all satellites in the receiver would not affect "normal" receiver operation, as they are entirely absorbed in the satellite clock solution during positioning. The slope has been removed empirically by fitting a first-order polynomial through the multipath combinations for the C/A-code below $30^{\circ}$ elevation. This correction has then been applied as correction not only to the C/A-code, but also to all other signals on L1 and L2.

Multipath characteristics for normal E-L correlators

We start the discussion with an overview of the results for the Javad DG3TH, the NovAtel OEMV, and the Septentrio PolaRx2 receivers using "conventional" early-late
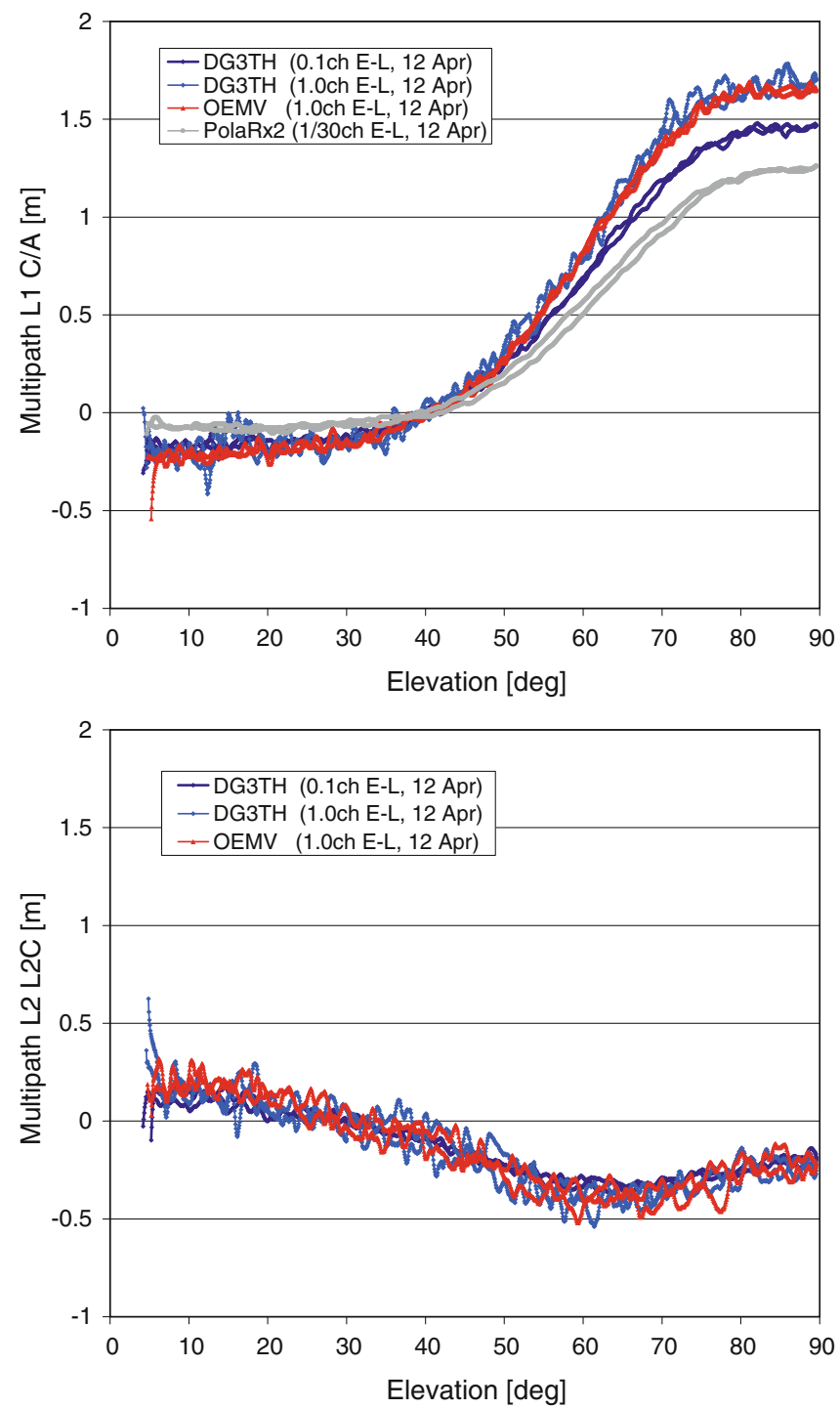

Fig. 3 Multipath over elevation for L1 C/A-code (upper left plot), L1 P-code (upper right plot), L2 C-code (lower left plot) and L2 P-code (lower right plot) for the Javad DG3TH, the NovAtel OEMV and the Septentrio PolaRx2 receiver for April 12, 2010. Note that the PolaRx2 correlators. The multipath combinations for different signals are shown in Fig. 3. All measurements stem from a satellite pass on April 12, 2010, with the exception of the OEMV measurements for P-code on L2, which were recorded on April 16. The two upper plots depict the multipath combination for C/A-code and P-code on L1. It becomes obvious that the C/A multipath curve starts with a negative offset of approximately $0.10 \mathrm{~m}$ for the PolaRx and $0.25 \mathrm{~m}$ for the other receivers. For elevations higher than $30^{\circ}$, the multipath curve starts to rise and reaches a maximum at zenith. It amounts to $1.7 \mathrm{~m}$ for the DG3TH and the OEMV with 1.0 chip correlator width. As expected, the multipath effect is smaller for smaller correlator spacing: for the DG3TH with 0.1 chip spacing, the maximum effect
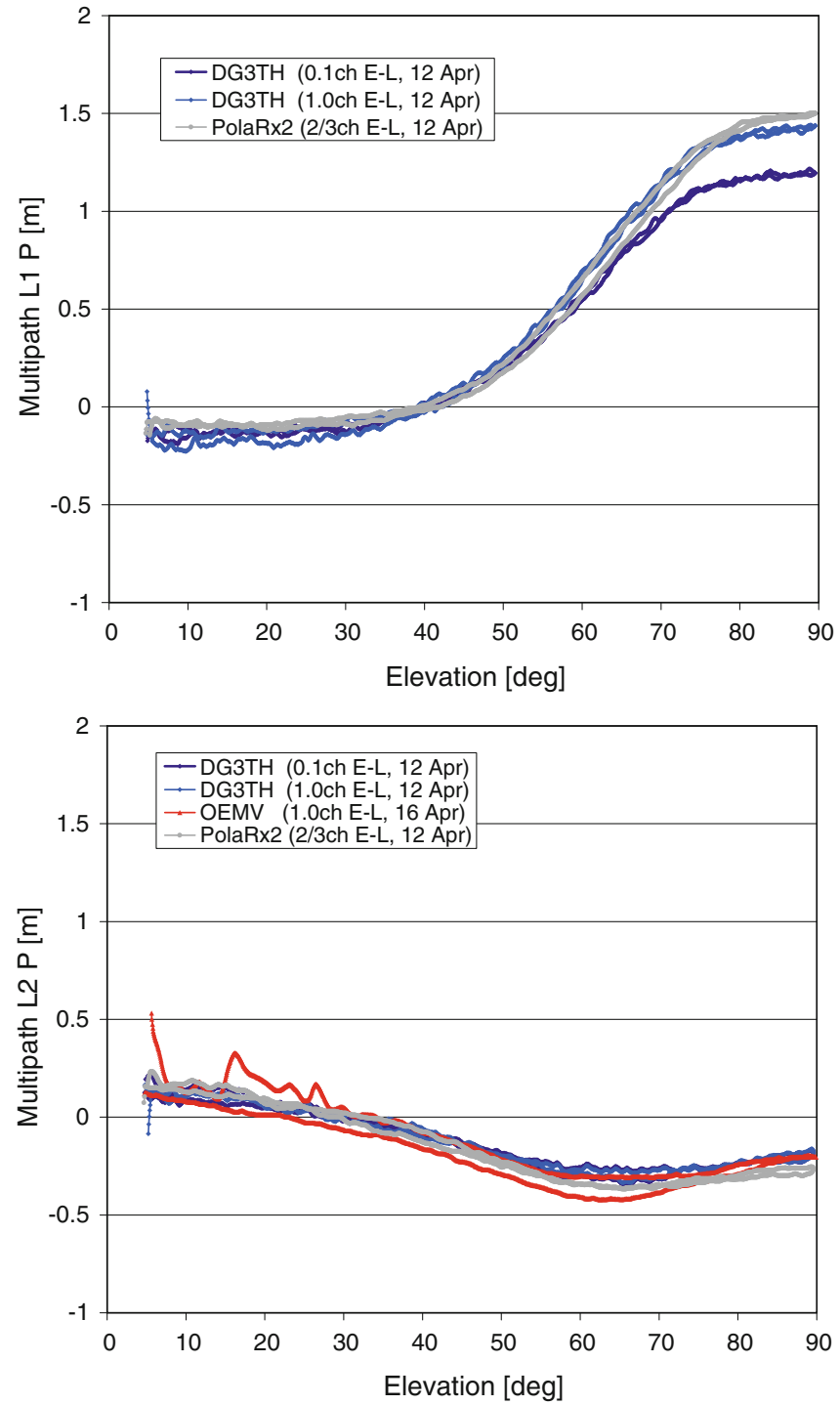

does not provide L2C measurements. The L2 P-code measurements from the OEMV stem from the pulse aperture correlator, which has been configured for tracking on April 16 
is $1.5 \mathrm{~m}$ and for the PolaRx2 with the smallest correlator width of $1 / 30$ of a chip, it amounts to only $1.25 \mathrm{~m}$. A similar picture can also be found for the P-code in the upper right plot. The DG3TH with 1.0 chip correlator spacing and the PolaRx2 with 0.6 chip spacing yield comparable results with $1.5 \mathrm{~m}$ for the maximum elevation. The DG3TH with 0.1 chip spacing exhibits a reduction in the reflection to $1.25 \mathrm{~m}$.

The plots for the signals on the L2 frequency show a significantly different elevation dependency. At low elevations, the lower left plot for the L2C signal starts at approximately $0.2 \mathrm{~m}$ for all receivers and reaches a minimum of about $-0.4 \mathrm{~m}$ at $65^{\circ}$ elevation. At zenith, the multipath reflection amounts to approximately $-0.25 \mathrm{~m}$. For the OEMV and the DG3TH with 1.0 chip correlator width, the L2C signal is significantly noisier than all other signals. A small reduction in the multipath effect can be recognized for the DG3TH receiver with the narrow correlator. The results for the P-code on L2 in the lower right plot do not differ significantly apart from the lower noise. However, the OEMV receiver exhibits a particular tracking behavior at low elevations. Note that the data for this receiver stems from April 16, when the PAC has been configured to track P-code signals. Closer review of the measurements from this day reveals a very similar tracking behavior of other receivers for P-code on L2 as well. This observation suggests that the unexpected variations are not caused by the OEMV receiver. A more likely explanation is signal interference on this particular day.

Having presented the effect of the signal reflection on different observables, its repeatability shall be discussed in the following. Figure 4 depicts the results of the multipath

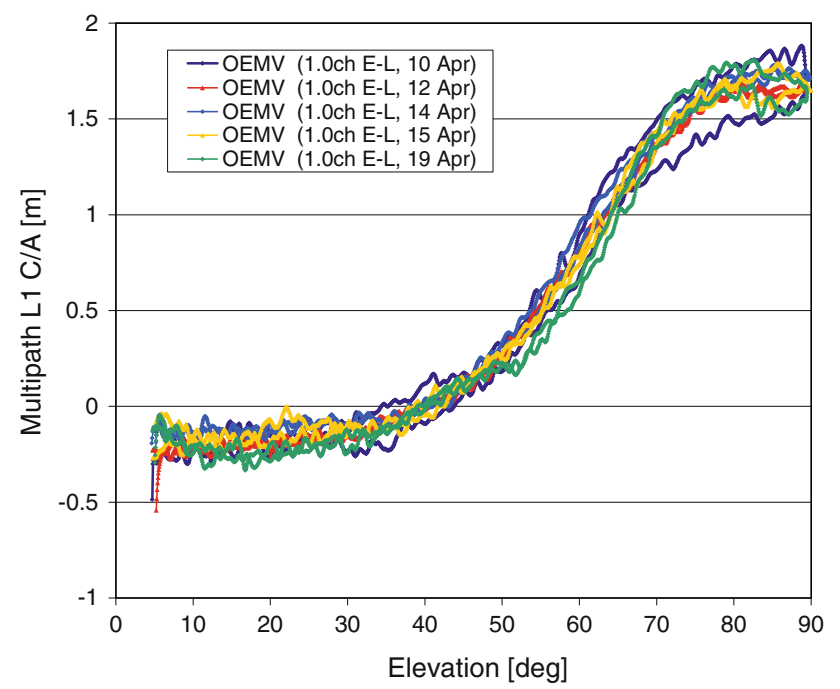

Fig. 4 Daily repeatability of the multipath effect over a period of 9 days from April 10 to April 19, 2010. The plot depicts the results for the multipath combination of the C/A-code signal for the OEMV receiver combination for the $\mathrm{C} / \mathrm{A}$-code for OEMV receiver for 5 days from April 10 until April 19. The plot shows a reasonable consistency for April 12, 14, and 15. All three curves are close together over the complete range of elevations. The plot for April 19 exhibits deviations especially at higher elevations where the ascending and descending part diverge. A similar effect but with larger magnitude can also be observed for the first day of the test interval. These differences in the multipath combination can either be a receiver-dependent or a satellite-dependent effect.

Overview of multipath mitigation methods

Finally, the results for correlators with special multipath mitigation feature shall be presented. Again, we limit the discussion to the results for the C/A-code signals. Figure 5 depicts results for all four receivers. Tracking data from the DG3TH, PolaRx2, and SF3050 receivers with multipath mitigation enabled is available for the satellite pass on April 19. The OEMV was operated with the PAC on April 16. It becomes obvious from the plot that the smallest mitigation effect is found for this correlator type. The maximum multipath at $90^{\circ}$ elevation still amounts to $1.5 \mathrm{~m}$, which is similar to 0.1 chip E-L correlator of the DG3TH. The PolaRx2 with the "A Posteriori Multipath Estimator" (APME) exhibits a better suppression of the signal reflection, however, at the price of a significant hysteresis effect. The ascending part of the curve is notably flatter than the descending part, and the maximum multipath error is shifted from zenith to approximately $80^{\circ}$ elevation. The divergence between the ascending and

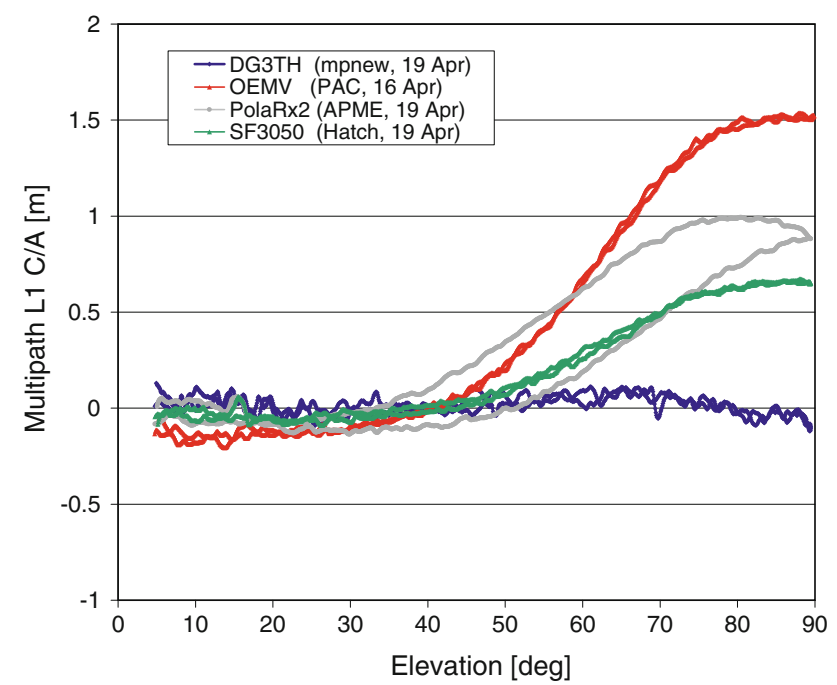

Fig. 5 Tracking results for C/A-code with special multipath mitigation techniques. The plot depicts tracking results for the Javad D3TH with "mpnew"-option, the NovAtel OEMV with PAC, the Septentrio PolaRx2 with APME option, and the NavCom SF3050 with Hatchcorrelator 
descending part of the pass is caused by the long time constant in the multipath estimator (Sleewagen, priv. comm.), but can also be expected if code-smoothing with a long time constant is applied. The second best mitigation is achieved with the Hatch-correlator of the SF3050 receiver. Due to its robustness toward multipath, it shows less than half of the maximum multipath error compared to a conventional 1.0-chip E-L correlator. Finally, the DG3TH receiver has been configured with a standard firmware version, which allows the selection of the "mpnew" multipath mitigation. This mitigation technique virtually eliminates the complete signal reflection, leaving only variations in the order of a decimeter. A similar performance can be expected with the OEMV Vision Correlator, which is further discussed in Part 2 of this study.

\section{Multipath characterization from IQ sample analysis}

After the presentation of the receiver tracking results, the analysis of the recorded IQ samples shall be presented. In this section, characteristic parameters like delay, attenuation, and phase shift of the reflected signal are determined, which will ultimately lead to a development of a complete model for the multipath. This method has been previously described in (Thoelert et al. 2009b) and is based on an iterative fit of simulated and measured IQ constellation diagrams. The left plot in Fig. 6 depicts normalized constellation diagrams for SVN49 at low elevation of $40^{\circ}$, where the reflected signal can be neglected. In direct comparison to the right plot, which shows the diagram at $89^{\circ}$ elevation, the change in the IQ constellation caused by multipath becomes clearly visible (Fig. 6).

In order to determine the characteristic parameters of the multipath, the measured samples used to generate the IQ constellation diagram at $40^{\circ}$ elevation are taken as a reference and merged with a replica of this signal, which has been attenuated, delayed in time, and shifted in phase. Then a constellation diagram is produced from the modified signal and correlated to the measured counterpart at high elevation. This procedure is repeated, and the attenuation, delay, and phase shift are varied over a predefined search space until the simulated constellation diagram with the highest correlation to the measured diagram is found (Thoelert et al. 2009b).

The plots in Fig. 7 show the estimation for delay $\tau$, attenuation $\alpha$, and phase shift $\varphi$ of the observed multipath of SVN49 for L1 and L2. The results are based on IQ samples of $5 \mathrm{~ms}$ length and taken every $10^{\circ}$ elevation. Measurements from April 14, 15, and 22, 2010 have been processed. For each day, the ascending and descending part
Fig. 6 IQ constellation diagrams for SVN49 at $40^{\circ}$ elevation (left) and $90^{\circ}$ elevation (right). The top plot depicts C/A- and P-code modulation on April 16, 2010, the bottom plot shows C/A-, $\mathrm{Y}-$, and $\mathrm{M}$-code modulation on April 22, 2010. The effect of the reflected signal is clearly visible in the distortion of the diagram for high elevations
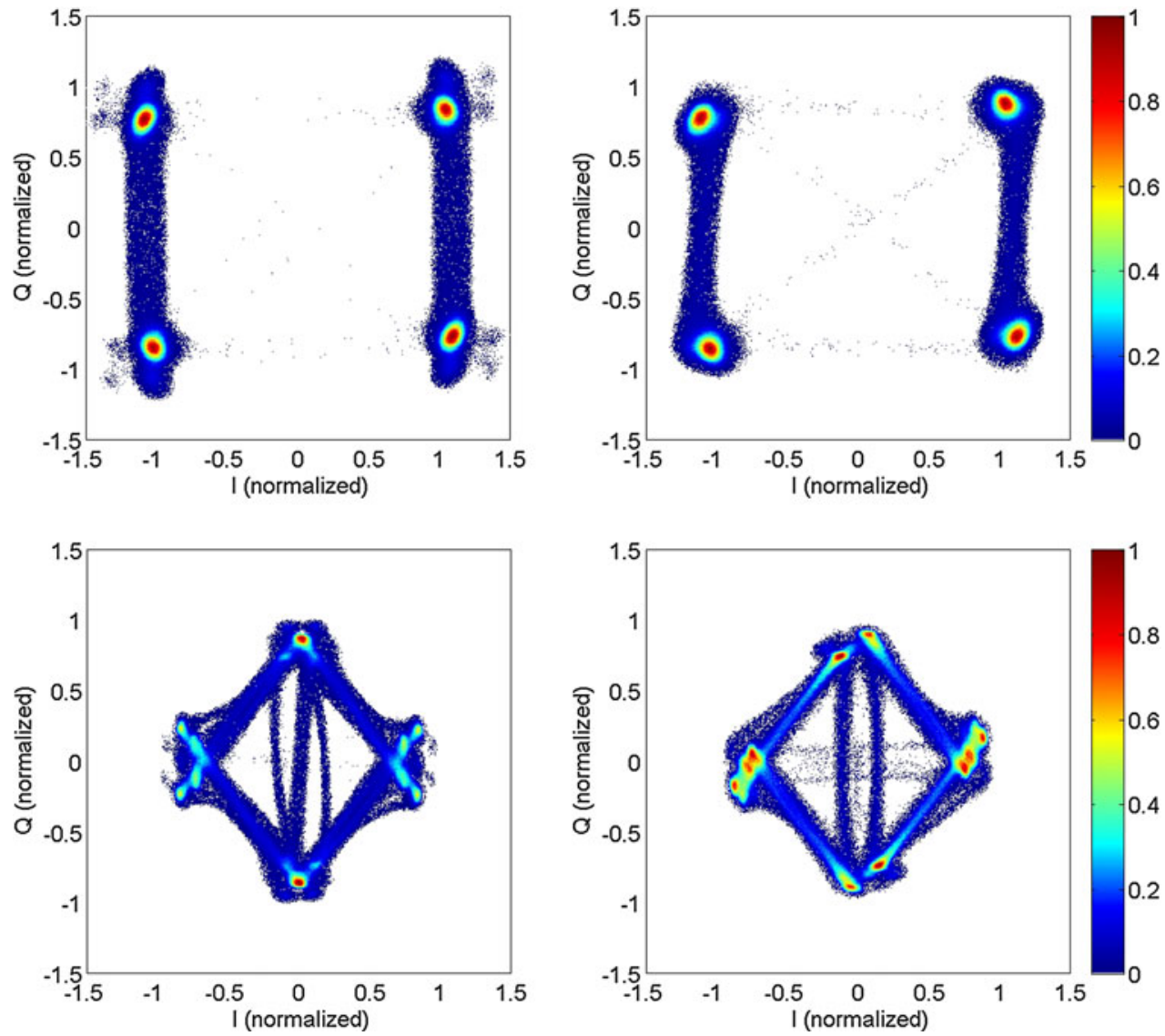

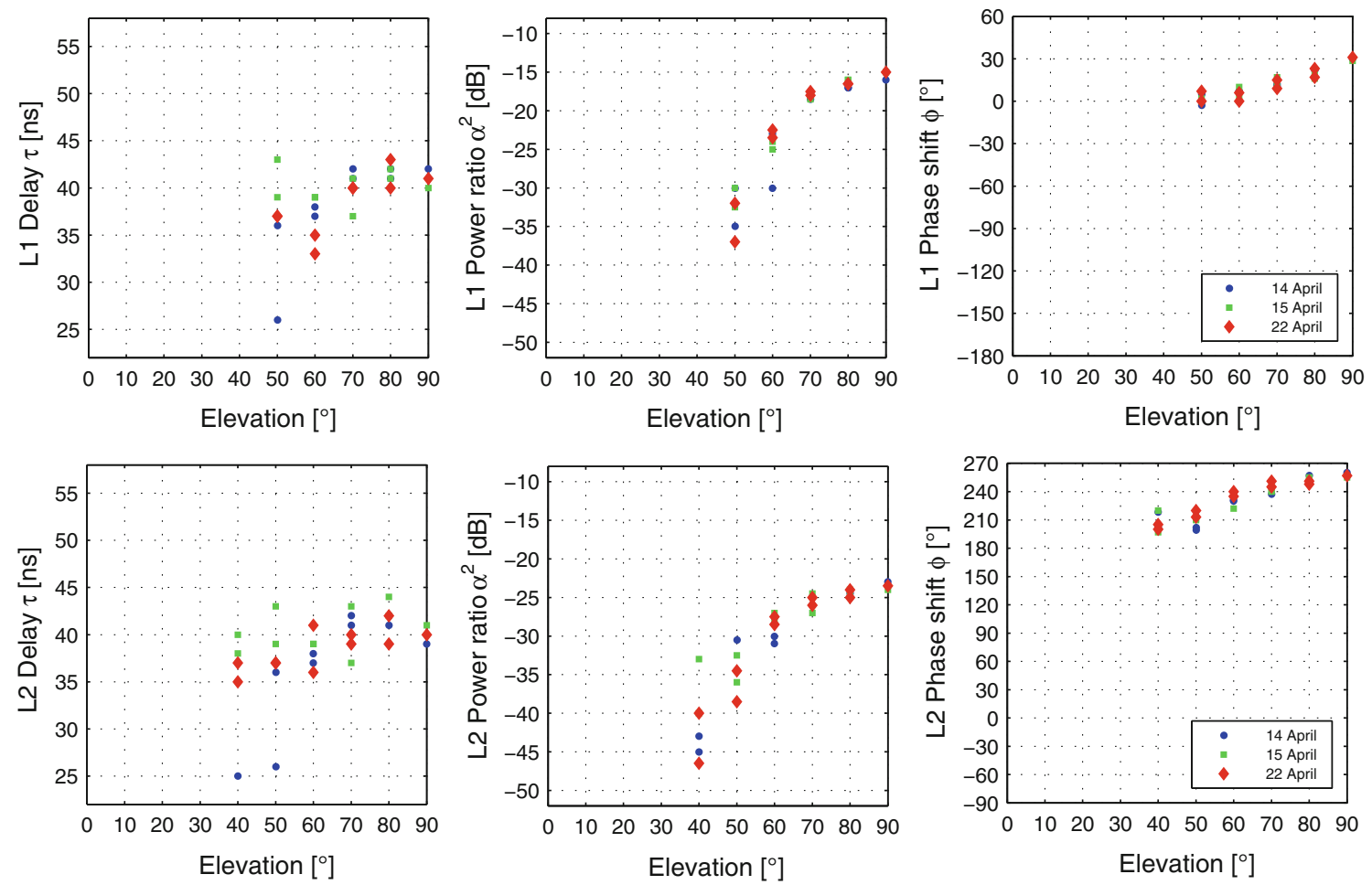

Fig. 7 SVN49 multipath parameters over elevation estimated based on IQ method for L1 (top plots) and L2 (bottom plots). Plots depict delay, attenuation (expressed as power ratio), and phase shift from left to right for April 14, 15, and 22, 2010

of the pass have been processed separately, resulting in two data points for each day. The estimated parameters exhibit a significant scatter, especially for lower elevations. This effect could be expected since the reflected signal is transmitted with lower power at low elevations and is thus more difficult to separate from noise and other errors. The plots are therefore confined to elevations above $40^{\circ}$ (L1) and $30^{\circ}$ (L2), which correspond to the nulls of the respective gain patterns for signals from the $\mathrm{J} 2$ port.

Based on the estimations at high elevation angles, the delay can be approximated to about $41 \mathrm{~ns}$ for $\mathrm{L} 1$ and $40 \mathrm{~ns}$ for L2. According to the multipath model in (6a), the delay should stay constant over the complete range of elevations. Instead, significant variations in the order of several tens of nanoseconds are visible for lower elevations on L1 and L2. The estimates of the power ratio yield consistent results for elevations higher than $60^{\circ}$. At zenith, the power ratio of the signal reflection is approximately $-15 \mathrm{~dB}$ on $\mathrm{L} 1$ and $-23 \mathrm{~dB}$ on L2. It varies over elevation governed by the different boresight-dependent gain patterns of the signals from the J1- and J2-port. The estimates of the phase shift exhibit only comparably little scatter over the entire range of elevations. At zenith, the L1 phase shift amounts to $30^{\circ}$. It varies over elevation due to the variation in the relative phase of the signal from the two antenna ports. At elevations lower than $30^{\circ}$, the maximum scatter between the different estimates is about $20^{\circ}$. For L2, the zenith phase shift is approximately $75^{\circ}$. The maximum scatter at low elevation is about $40^{\circ}$.

Based on the relative amplitude $\alpha$ and phase shift $\varphi$ of the reflected and direct signal derived from the IQ constellation diagrams, an estimate of the carrier phase tracking error

$\Delta \Phi=\frac{1}{2 \pi} \frac{\alpha \sin \varphi}{1+\alpha \cos \varphi}$

(in cycles) may be derived from the respective phase vectors (Braasch 1996). Near zenith, a carrier phase error of about $2.5 \mathrm{~mm}$ is obtained for both $\mathrm{L} 1 \quad(\alpha \approx 0.18$, $\left.\varphi \approx 30^{\circ}\right)$ and L2 signals $\left(\alpha \approx 0.07, \varphi \approx 260^{\circ}\right.$ ). The total effect on the ionosphere-free L1/L2 carrier phase combination is thus less than $1 \mathrm{~cm}$ and potentially masked by local site multipath in geodetic reference stations. It may be noted, though, that systematic carrier phase errors, which can be attributed to the SVN49 internal multipath, have recently been identified in an analysis of GPS tracking data from low Earth satellites (Jäggi et al. 2010).

\section{Summary and conclusions}

We provided an overview of the tracking campaign of SVN49 for an analysis of the satellite's internal signal reflection. DLR's 30-m dish antenna has been used to track passes with elevations higher than $89^{\circ}$. Measurements have 
been collected with a set of receivers to assess the impact of the satellite's internal signal reflection on the tracking using different correlator implementations. Due to the high directivity of the deep space antenna, local multipath errors are virtually eliminated and the high antenna gain allows tracking with high signal-to-noise ratios over the entire range of elevations from less than $5^{\circ}$ up to zenith. The multipath combination of signals on different frequencies has been analyzed. For C/A-code tracking on L1 with conventional early-minus-late correlators with 1.0 chip spacing, the multipath error ranges from $-0.25 \mathrm{~m}$ at $5^{\circ}$ elevation to $1.7 \mathrm{~m}$ at zenith, when normalizing the curve to zero at $40^{\circ}$ elevation. For L2C code, the plot of the multipath combination over elevation has a different shape. It starts at about $0.2 \mathrm{~m}$ for low elevations, reaches $-0.4 \mathrm{~m}$ for $65^{\circ}$ elevation, and amounts to $-0.25 \mathrm{~m}$ at zenith. As expected, the impact of the signal reflection is reduced for narrow correlators. The repeatability of the results has been analyzed using tracking data from five satellite passes. Whereas the passes of the three middle days are consistent, the first and the last day of the test period exhibit notable deviations.

Complementary results are obtained from a technique to determine delay, phase shift, and attenuation of the reflection based on an iterative correlation of measured and simulated IQ constellation diagrams. As a first approximation, the reflected signal on L1 is delayed by $41 \mathrm{~ns}$. At zenith, the observed multipath is attenuated by $-15 \mathrm{~dB}$ and shifted by $30^{\circ}$ in phase. For L2, the delay amounts to about $40 \mathrm{~ns}$ and the power ratio and phase shift at zenith are $-23 \mathrm{~dB}$ and $75^{\circ}$, respectively.

The companion paper will present results for the chip shape reconstructed from the IQ samples and from vision correlator measurements with the OEMV receiver. Finally, a multipath model will be derived to simulate the results for the different early-minus-late correlators obtained from the receiver tracking.

Acknowledgments The authors would like to thank the GPS Wing, Aerospace Corporation, and Stansell Consulting Inc. for fruitful discussions and technical support in setting up the SVN49 test campaign. We are also grateful for the vital support of the Weilheim operations team that has notably contributed to the successful performance of the SVN49 tracking. Receivers and test-specific firmware modifications for the data collection have been provided by Javad GNSS, NavCom, and NovAtel. The support of all manufacturers is equally acknowledged.

Open Access This article is distributed under the terms of the Creative Commons Attribution Noncommercial License which permits any noncommercial use, distribution, and reproduction in any medium, provided the original author(s) and source are credited.

\section{References}

Braasch M (1996) Multipath effects. In: GPS positioning system theory and applications vol 1. American Institute of Aeronautics and Astronautics, pp 547-568

El-Arini MB, Conker RS, O'Laughlin DG, Hegarty CJ (2010) Effect of SVN49 (PRN01) on position and time accuracy for single-and dual-frequency users. In: Proceedings of ION-GNSS-2010, 21-24 Sept 2010, Portland, OR, USA

Ericson S, Shallberg K, Edgar C (2010) Characterization and simulation of SVN49 (PRN01) elevation dependent measurement biases. In: Proceedings of ION ITM 2010, 25-27 Jan 2010, San Diego, CA, USA

Erwin B, Irvine J, McFadden M, Powell T, Marquis W (2009) L5 demo payload. GPS World, May 2009, pp 27-31

Gao GX, Heng L, De Lorenzo D, Lo S, Akos D, Chen A, Walter T, Enge P, Parkinson B (2009) Modernization milestone-observing the first GPS satellite with an L5 payload. Inside GNSS, May/June 2009, pp 30-36

Hatch R, Knight J, Dai L (2007) Recent GNSS developments at NavCom technology. In: Proceedings of international global navigation satellite systems society (IGNSS) symposium, 4-6 Dec 2007, Sydney, Australia

Jäggi A, Dach R, Bock H, Beutler G, Montenbruck O, Schmid R (2010) Extending the GPS satellite antenna patterns of the IGS to nadir angles beyond $14^{\circ}$ using LEO data. AGU fall meeting 2010, 13-17 Dec 2010, San Francisco, California

Jones J, Fenton P, Smith B (2004) Theory and performance of the pulse aperture correlator. Available at http://www.novatel.com/ Documents/Papers/PAC.pdf

Kee C, Parkinson B (1994) Calibration of multipath errors on GPS pseudorange measurements. In: Proceedings of the 7th international technical meeting of the satellite division of the institute of navigation, Salt Lake City, UT, Sept 20-23 1994, pp 353-362

Komjathy A, Wilson BD, Mannucci AJ (2010) New developments on estimating satellite interfrequency bias for SVN49. GPS solutions. doi:10.1007/s10291-010-0185-5

Lake J, Stansell T (2009); SVN-49 signal anomaly; presented at 49th meeting of the civil GPS service interface committee (CGSIC), Savannah, GA, USA

Langley RB (2009) Expert advice: cause identified for pseudorange error from new GPS satellite SVN49. GPS world, August 2009, pp 8-12

Meurer M, Erker S, Thoelert S, Montenbruck O, Hauschild A, Langley RB (2009) GPS L5 First Light, GPS World, June 2009, pp 49-59

Sleewaegen JM, Boom F (2001) Mitigating short-delay multipath: a promising new technique, proceedings of ION GPS 2001, 11-14 Sept 2001, Salt Lake City, UT, USA

Springer T, Dilssner F (2009) SVN49 and other GPS anomalies, Inside GNSS July/August 2009, pp 32-36

Thoelert S, Erker S, Montenbruck O, Hauschild A, Meurer M (2009a) GPS SVN49-L1 anomaly analysis based on measurements with a high gain antenna, 4th European workshop on GNSS signals and signal processing, Oberpfaffenhofen

Thoelert S, Erker S, Meuer M (2009b) GNSS signal verification with a high gain antenna - calibration strategies and high quality signal assessment; ION ITM 2009, 26-28 Jan 2009, Anaheim, CA 


\section{Author Biographies}
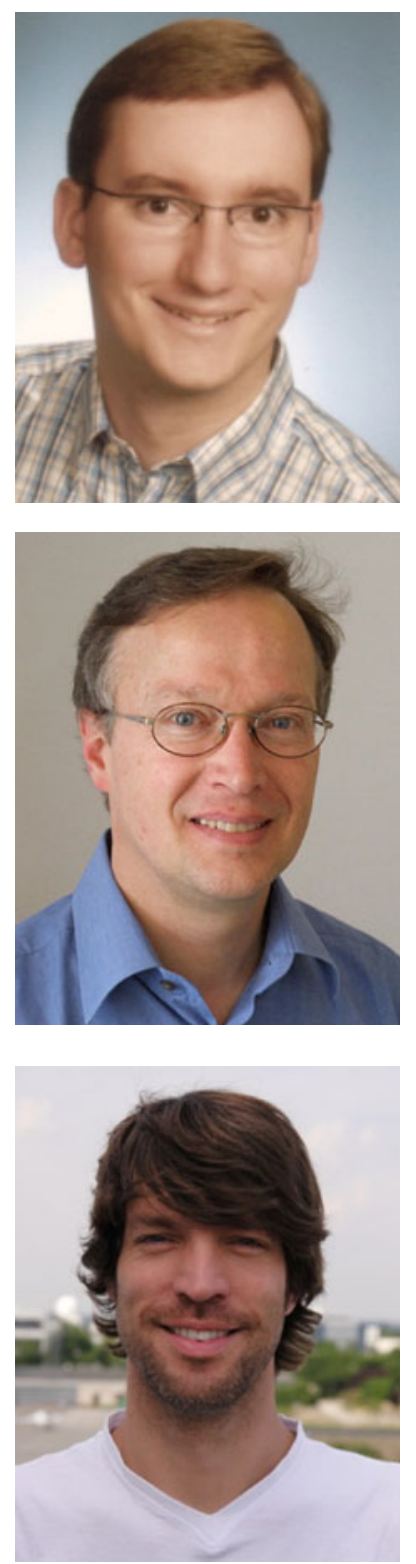

Dr. André Hauschild is a scientific staff member at DLR's German Space Operations Center (GSOC). His field of work focuses on precise real-time orbit and clock estimation for GNSS satellites as well as multi-GNSS processing using modernized GPS and new satellite navigation systems.

Dr. Oliver Montenbruck is head of the GNSS Technology and Navigation Group at DLR's German Space Operations Center, where he started to work as a flight dynamics analyst in 1987. His current research activities comprise spaceborne GNSS receiver technology, autonomous navigation systems, spacecraft formation flying, precise orbit determination, and multiconstellation GNSS.

Steffen Thoelert received his diploma degree in Electrical Engineering at the University of Magdeburg, Germany, in 2002. Since then he has been a member of the scientific staff at the German Aerospace Centre (DLR). In 2006 he joined the Institute of Communications and Navigation at the German Aerospace Centre (DLR). His current work is in the fields of GNSS verification and system calibration.

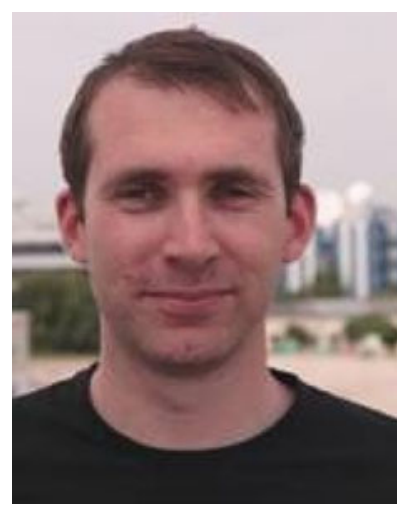

Stefan Erker received his diploma degree in Communication Technology at the Technical University of Kaiserslautern, Germany in 2007. In the same year he joined the Institute of Communications and Navigation of the German Aerospace Centre (DLR) at Oberpfaffenhofen. He mainly works on the topics of GNSS verification and corresponding signal analysis.

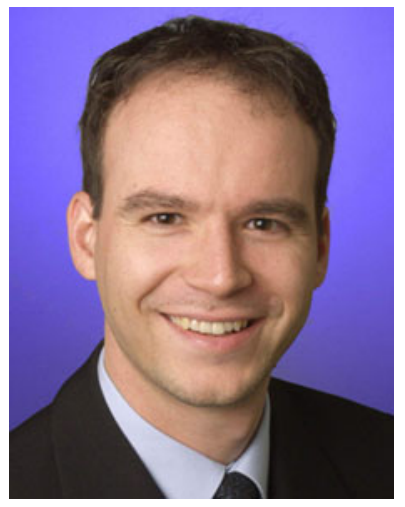

Dr. Michael Meurer is head of the Department of Navigation at DLR's Institute for Communications and Navigation, and coordinating director of the DLR Centre of Excellence for Satellite Navigation. Since 2005 he has been an Associate Professor (PD) at the Technical University of Kaiserslautern. His research interests include GNSS signals, GNSS receivers, interference mitigation and navigation for safety-critical applications.

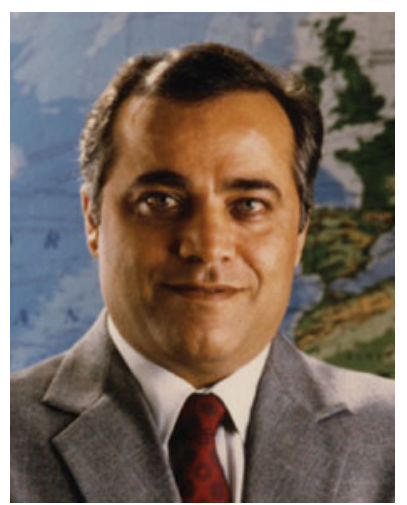

Dr. Javad Ashjaee received a B.S. in Electronics Physics from University of Tehran, M.S. degrees in Mathematics and in Electrical Engineering, as well as a Ph.D. in Electrical Engineering from University of Iowa (1986). He copioneered highprecision GPS technology at Trimble Navigation (1981 to 1986), founded Ashtech (1986), Javad Positioning Systems (1996) and Javad GNSS (2005) in pursuit of GNSS technology innovations. 